\title{
Optimal control of a population dynamics model with hysteresis *
}

\author{
Sergey A. Timoshin ${ }^{\dagger \ddagger}$ and Chen Bin ${ }^{\S}$
}

\begin{abstract}
This paper addresses a nonlinear partial differential control system arising in population dynamics. The system consist of three diffusion equations describing the evolutions of three biological species: prey, predator, and food for the prey or vegetation. The equation for the food density incorporates a hysteresis operator of generalized stop type accounting for underlying hysteresis effects occurring in the dynamical process. We study the problem of minimization of a given integral cost functional over solutions of the above system. The set-valued mapping defining the control constraint is state-dependent and its values are nonconvex as is the cost integrand as a function of the control variable. Some relaxation-type results for the minimization problem are obtained and the existence of a nearly optimal solution is established.
\end{abstract}

Keywords: optimal control problem; hysteresis; biological diffusion models; nonconvex integrands; nonconvex control constraints.

\footnotetext{
${ }^{*}$ Supported by National Natural Science Foundation of China (12071165 and 62076104), Natural Science Foundation of Fujian Province (2020J01072), Program for Innovative Research Team in Science and Technology in Fujian Province University, Quanzhou High-Level Talents Support Plan (2017ZT012), and by Scientific Research Funds of Huaqiao University (605-50Y19017, 605-50Y14040). The research of the first author was also supported by Ministry of Science and Higher Education of Russian Federation (Grant No. 075-15-2020-787, large scientific project "Fundamentals, methods and technologies for digital monitoring and forecasting of the environmental situation on the Baikal natural territory").

${ }^{\dagger}$ Fujian Province University Key Laboratory of Computational Science, School of Mathematical Sciences, Huaqiao University, Quanzhou 362021, China - and - Matrosov Institute for System Dynamics and Control Theory, Russian Academy of Sciences, Lermontov str. 134, 664033 Irkutsk, Russia, E-mail: sergey.timoshin@gmail.com.

${ }^{\ddagger}$ Corresponding author.

${ }^{\S}$ Fujian Province University Key Laboratory of Computational Science, School of Mathematical Sciences, Huaqiao University, Quanzhou 362021, China, E-mail: chenbinmath@163.com.
} 
2010 Mathematics Subject Classification: 49J20, 49J21, 49J45, 49J53.

Abbreviated title: Optimal control of a biomodel with hysteresis.

\section{Introduction}

Given a final time $T>0$ and a bounded domain $\Omega \subset \mathbb{R}^{N}, N \leq 3$, with smooth boundary $\partial \Omega$, the primary concern of this paper is to consider the following optimal control problem:

$$
J(\sigma, v, w, u):=\int_{0}^{T} \int_{\Omega} q(t, x, \sigma(t, x), v(t, x), w(t, x), u(t, x)) d x d t \rightarrow \inf
$$

over the solution set of the dynamical control system:

$$
\begin{gathered}
\sigma_{t}-(\lambda(v))_{t}-\kappa \Delta \sigma+\partial I_{v, w}(\sigma) \ni F(\sigma, v, w) u \quad \text { in } Q(T), \\
v_{t}-\Delta v=h(\sigma, v, w) \quad \text { in } Q(T), \\
w_{t}-\Delta w=g(\sigma, v, w) \quad \text { in } Q(T), \\
\sigma(x, 0)=\sigma_{0}(x), \quad v(x, 0)=v_{0}(x), \quad w(x, 0)=w_{0}(x) \quad \text { in } \Omega, \\
\frac{\partial \sigma}{\partial n}=\frac{\partial v}{\partial n}=\frac{\partial w}{\partial n}=0 \quad \text { on } \partial \Omega \times[0, T],
\end{gathered}
$$

subject to the state-dependent control constraint:

$$
u \in U(t, x, \sigma, v, w) \quad \text { in } Q(T) .
$$

Here, $Q(T):=[0, T] \times \Omega, \kappa$ is a given constant, $\sigma_{0}, v_{0}, w_{0}$ are given initial conditions, $\partial / \partial n$ is the outward normal derivative on $\partial \Omega$. The operator $\partial I_{v, w}(\cdot)$ is the subdifferential, in the sense of convex analysis, of the indicator function $I_{v, w}(\cdot)$ of the interval $\left[f_{*}(v, w), f^{*}(v, w)\right], f_{*}, f^{*}: \mathbb{R}^{2} \rightarrow \mathbb{R}, q: Q(T) \times \mathbb{R}^{4} \rightarrow \mathbb{R}, F, h, g: \mathbb{R}^{3} \rightarrow \mathbb{R}$, $\lambda: \mathbb{R} \rightarrow \mathbb{R}$ are prescribed functions, $U: Q(T) \times \mathbb{R}^{3} \rightarrow \mathbb{R}$ is a multivalued mapping with compact, but not necessarily convex, values.

We note that when $w$ is fixed and $F \equiv 0, \lambda(v)=v$ in (1.1), inclusion (1.1) yields the differential representation of hysteresis operator of the generalized stop type (cf. [1]). During the last three decades, hysteresis operators have been extensively applied in modeling, analysis, and control of a variety of irreversible nonlinear phenomena in applied sciences including phase transitions [2 7], porous media flow [8] 12, thermostat models 13 17, concrete carbonation [18 22] and many others. In the same vein, system (1.1)-(1.5) (without the control $u$ ) was introduced in [23] (see, also, 24, 25]) to model the evolution of populations in the vegetation-preypredator framework when diffusive effects in the dynamics of three species are taken into account and the food density for the prey exhibits hysteretic character. This latter means that the growth rate of the food for the prey depends not only on the current state of prey and predator populations, but it also depends on their 
immediately preceding density history. In [26, with the aim to achieve a possible optimization of the population dynamics process by way of controlling the food supply for the prey this uncontrolled system was subjected to a control action and the existence of solutions for the corresponding control system (1.1)-(1.6) was established.

In our model, the unknown variables $\sigma, v$, and $w$ represent the densities of vegetation, prey and predator, respectively, with Eqs. (1.1), (1.2), and (1.3) characterizing the evolutions of the corresponding species. In this respect, the nonconvexity of values of $U$ in the control constraint (1.6) is a biologically relevant assumption.

Our aim in this paper is to consider along with $(P)$ the following alternative problem:

$$
J_{U}^{* *}(\sigma, v, w, u):=\int_{0}^{T} \int_{\Omega} q_{U}^{* *}(t, x, \sigma(t, x), v(t, x), w(t, x), u(t, x)) d x d t \rightarrow \inf
$$

over solutions of (1.1)-(1.5) supplemented with the following alternative control constraint:

$$
u \in \operatorname{co} U(t, x, \sigma, v, w) \quad \text { in } Q(T),
$$

and explore some properties and relationships between Problems $(P)$ and $(R P)$. Here, $(R P)$ stands for the relaxed problem, co $U$ denotes the convex hull of the set $U$, which is the intersection of all convex sets containing $U$, and for the extended real-valued function $q_{U}: Q(T) \times \mathbb{R}^{3} \times \mathbb{R} \rightarrow \mathbb{R} \cup\{+\infty\}:$

$$
q_{U}(t, x, \sigma, v, w, u)= \begin{cases}q(t, x, \sigma, v, w, u) & \text { if } u \in U(t, x, \sigma, v, w), \\ +\infty & \text { otherwise, }\end{cases}
$$

$q_{U}^{* *}(t, x, \sigma, v, w, u)$ is the bipolar or the second conjugate of the function $u \mapsto$ $q_{U}(t, x, \sigma, v, w, u)$, which is the largest lower semicontinuous convex function less than or equal to $q_{U}^{* *}$. We note that both the values of the set-mapping in the control constraint and the integrand as a function of control in Problem $(R P)$ are convex.

We remark that while our model has not been inspired by any particular applied problem, the results we obtain might find potential applications in analysis and control of real-world ecosystems such as spruce budworm population dynamics models. The latter describe interactions in the budworm-forest framework and include three species: boreal forest trees such as balsam fir, insect pests such as spruce budworm which feed on the foliage of trees and avian predators feeding in turn on the insects. These ecosystems are typical to Northern parts of Canada and Russia, including the lake Baikal region, where the defoliation of larches by insects and the subsequent dieback of the trees is a major problem in forest ecology. Available control actions towards preservation of forest reserve include spraying insecticides, removal of infected trees and other and affect the rate of change in the insects population. This prompted us to include the control function $u$ into the first equation of our evolution system. On the other hand, the natural concern to avoid the total perishing of birds population caused by insufficient food supply should be reflected in optimization strategy towards minimization of forest loss. 
The outline of the paper is as follows. After introducing in the next section the notation and hypotheses on the data describing Problem $(P)$, in Section 3 we derive several continuity properties of the control-to-state solution operator associated with our control systems. These properties are instrumental in proving our main results in the last Section 4. The latter consist in establishing the existence of an optimal solution for Problem $(R P)$, the density, in an appropriate topology, of solutions of the original control system (1.1)-(1.6) among solutions of the convexified control system (1.1)-(1.5), (1.7), and the existence of a nearly optimal, in a suitable sense, solution for problem $(P)$.

At the end of Introduction, we mention that the existing literature on optimal control of systems exhibiting hysteretic character is sparse. It is worthwhile however to mention a seminal contribution of M. Brokate to this area (see 2728]). Moreover, to the best of the authors' knowledge, there have been no contributions so far addressing optimal control problems for biological models with hysteresis.

Acknowledgements. The authors want to thank the anonymous referees for their valuable suggestions and remarks which helped to improve the manuscript.

\section{Preliminary notions, hypotheses on the data, and statement of the main results}

In this section, we fix the notation which we use throughout the paper, prove an auxiliary lemma, specify hypotheses which we posit on the data describing Problem $(P)$, give a precise meaning in which solutions to our control systems are understood and the corresponding minimization problems are treated, and state our main results.

Let $X$ be a Hilbert space with the inner product $(\cdot, \cdot)_{X}$. A function $\varphi: X \rightarrow$ $\mathbb{R} \cup\{+\infty\}$ is called proper if its effective domain $\operatorname{dom} \varphi:=\{x \in X ; \varphi(x)<+\infty\}$ is nonempty. By definition, the subdifferential $\partial \varphi(x), x \in X$, of a proper, convex, lower semicontinuous function $\varphi$ is the set

$$
\partial \varphi(x)=\left\{h \in X ;(h, y-x)_{X} \leq \varphi(y)-\varphi(x), \forall y \in X\right\},
$$

and its domain is the set $\operatorname{dom} \partial \varphi:=\{x \in X ; \partial \varphi(x) \neq \emptyset\}$. It is known 29] that $\operatorname{dom} \partial \varphi \subset \operatorname{dom} \varphi$.

We say that a sequence of proper, convex, lower semicontinuous functions $\varphi_{n}: X \rightarrow \mathbb{R}, n \geq 1$, Mosco-converges [30] to a proper, convex, lower semicontinuous function $\varphi: X \rightarrow \mathbb{R}$, denoted $\varphi_{n} \stackrel{M}{\rightarrow} \varphi$, if:

1) for any $x \in X$ and any sequence $x_{n} \in X, n \geq 1$, weakly converging to $x$ we have

$$
\varphi(x) \leq \liminf _{n \rightarrow \infty} \varphi_{n}\left(x_{n}\right) ;
$$

2) for any $x \in X$ there exists a sequence $x_{n} \rightarrow x$ such that $\varphi_{n}\left(x_{n}\right) \rightarrow \varphi(x)$. 
Denote by $d_{X}(x, A)$ the distance from a point $x \in X$ to a set $A \subset X$. Then, the Hausdorff metric on the space of closed bounded subsets of $X$, denoted $c b(X)$, is the function:

$$
\operatorname{haus}_{X}(A, B)=\max \left\{\sup _{x \in A} d_{X}(x, B), \sup _{y \in B} d_{X}(y, A)\right\}, \quad A, B \in c b(X) .
$$

If $(\mathcal{E}, \mathcal{A})$ is a measurable space, then a multivalued mapping $F: \mathcal{E} \rightarrow c b(X)$ is called measurable if $\{\tau \in \mathcal{E} ; F(\tau) \cap C \neq \emptyset\} \in \mathcal{A}$ for any closed subset $C$ of $X$. A set $\mathcal{F}$ of measurable functions from $\mathcal{E}$ to $X$ is called decomposable if for any $f_{1}, f_{2} \in \mathcal{F}$ and any $E \in \mathcal{A}$ we have that $f_{1} \cdot \chi_{E}+f_{2} \cdot \chi_{\mathcal{E} \backslash E} \in \mathcal{F}$, where $\chi_{E}$ stands for the characteristic function of the set $E$.

Let $Y, Z$ be two Banach spaces. A multivalued mapping $G: Y \rightarrow Z$ is called lower semicontinuous if for any $y \in Y, z \in F(y)$ and any sequence $y_{k} \rightarrow y, k \geq 1$, there exists a sequence $z_{k} \in F\left(y_{k}\right), k \geq 1$, which converges to $z$.

Given two functions $f_{*}, f^{*}: \mathbb{R}^{2} \rightarrow \mathbb{R}$, recall that the indicator function $I_{v, w}(\cdot)$ of the set $K(v, w):=\left[f_{*}(v, w), f^{*}(v, w)\right]$ is defined as follows

$$
I_{v, w}(\sigma):=\left\{\begin{array}{cl}
0 & \text { if } \sigma \in K(v, w) \\
+\infty & \text { otherwise. }
\end{array}\right.
$$

Its subdifferential has the form:

$$
\partial I_{v, w}(\sigma)=\left\{\begin{array}{cll}
\emptyset & \text { if } \sigma \notin K(v, w), \\
{[0,+\infty)} & \text { if } \sigma=f^{*}(v, w)>f_{*}(v, w), \\
\{0\} & \text { if } f_{*}(v, w)<\sigma<f^{*}(v, w), \\
(-\infty, 0] & \text { if } \sigma=f_{*}(v, w)<f^{*}(v, w), \\
(-\infty,+\infty) & \text { if } \sigma=f_{*}(v, w)=f^{*}(v, w) .
\end{array}\right.
$$

And, for $\mu>0$, the Yosida regularization of $\partial I_{v, w}(\sigma)$ is the function

$$
\partial I_{v, w}^{\mu}(\sigma)=\frac{1}{\mu}\left[\sigma-f^{*}(v, w)\right]^{+}-\frac{1}{\mu}\left[f_{*}(v, w)-\sigma\right]^{+}, \quad \sigma, v, w \in \mathbb{R} .
$$

Let $H$ be a Hilbert space with the norm $|\cdot|_{H}$.

Lemma 2.1. Let $v_{n} \rightarrow v, w_{n} \rightarrow w, \sigma_{n} \rightarrow \sigma$ in $L^{2}(0, T ; H), f_{n} \rightarrow f$ weakly in $L^{2}(0, T ; H)$. In addition, assume that a set-valued function $\mathcal{K}: H \times H \rightarrow c b(H)$ has convex values and

$$
\operatorname{haus}_{H}\left(\mathcal{K}\left(v_{1}, w_{1}\right), \mathcal{K}\left(v_{2}, w_{2}\right)\right) \leq R\left(\left|v_{1}-v_{2}\right|_{H}+\left|w_{1}-w_{2}\right|_{H}\right),
$$

$v_{i}, w_{i} \in H, i=1,2$, where $R>0$ is a constant. If

$$
f_{n}(t) \in \partial I_{\mathcal{K}\left(v_{n}(t), w_{n}(t)\right)}\left(\sigma_{n}(t)\right) \quad \text { for a.e. } t \in[0, T]
$$

then

$$
f(t) \in \partial I_{\mathcal{K}(v(t), w(t))}(\sigma(t)) \quad \text { for a.e. } t \in[0, T],
$$

where $I_{\mathcal{K}(v(t), w(t))}$ is the indicator function of the set $\mathcal{K}(v(t), w(t)) \subset H, t \in[0, T]$. 
Proof. First, we define on $L^{2}(0, T ; H)$ the function

$$
I_{\mathcal{K}(v, w)}(\sigma)=\int_{0}^{T} I_{\mathcal{K}(v(\tau), w(\tau))}(\sigma(\tau)) d \tau
$$

It is easy to see that $I_{\mathcal{K}(v, w)}$ is the indicator function of the set

$$
\mathcal{K}(v, w)=\left\{\sigma \in L^{2}(0, T ; H) ; \sigma(t) \in \mathcal{K}(v(t), w(t)) \text { a.e. on }[0, T]\right\}
$$

and it is proper, convex, and lower semicontinuous. Moreover, 31, Proposition 0.3.3] implies that

$$
f_{n} \in \partial I_{\mathcal{K}\left(v_{n}, w_{n}\right)}\left(\sigma_{n}\right), \quad n \geq 1 .
$$

Next, we prove that $I_{\mathcal{K}\left(v_{n}, w_{n}\right)} \stackrel{M}{\rightarrow} I_{\mathcal{K}(v, w)}$. According to the definition of the Mosco-convergence introduced above, we need to show that for any sequence $z_{n} \rightarrow z$ weakly in $L^{2}(0, T ; H)$ we have

$$
I_{\mathcal{K}(v, w)}(z) \leq \liminf _{n \rightarrow \infty} I_{\mathcal{K}\left(v_{n}, w_{n}\right)}\left(z_{n}\right),
$$

and for any $z \in L^{2}(0, T ; H)$ there exists a sequence $z_{n} \rightarrow z$ in $L^{2}(0, T ; H)$ such that

$$
I_{\mathcal{K}\left(v_{n}, w_{n}\right)}\left(z_{n}\right) \rightarrow I_{\mathcal{K}(v, w)}(z)
$$

So, let $z_{n} \rightarrow z$ weakly in $L^{2}(0, T ; H)$. In the case when $\liminf _{n \rightarrow \infty} I_{\mathcal{K}\left(v_{n}, w_{n}\right)}\left(z_{n}\right)=$ $+\infty$, (2.3) trivially holds. Hence, without loss of generality, we can assume that $I_{\mathcal{K}\left(v_{n}, w_{n}\right)}\left(z_{n}\right)=0, n \geq 1$, which implies that $z_{n} \in \mathcal{K}\left(v_{n}, w_{n}\right)$. Invoking the Mazur lemma from this inclusion and (2.1) we infer that

$$
z \in \bigcap_{k=1}^{\infty} \overline{\mathrm{co}}\left(\bigcup_{n=k}^{\infty} z_{n}\right) \subset \overline{\mathrm{co}} \mathcal{K}(v, w)=\mathcal{K}(v, w) .
$$

Therefore, $I_{\mathcal{K}(v, w)}(z)=0$ and (2.3) follows.

Take now an arbitrary $z \in L^{2}(0, T ; H)$. When $I_{\mathcal{K}(v, w)}(z)=+\infty$, (2.3) implies that (2.4) holds for any sequence $z_{n} \rightarrow z$ in $L^{2}(0, T ; H)$. Consequently, we assume that $I_{\mathcal{K}(v, w)}(z)=0$, i.e. $z \in \mathcal{K}(v, w)$. From (2.1) it follows that we can find a sequence $z_{n} \rightarrow z$ in $L^{2}(0, T ; H), z_{n} \in \mathcal{K}\left(v_{n}, w_{n}\right)$. In this case, $I_{\mathcal{K}\left(v_{n}, w_{n}\right)}\left(z_{n}\right)=0$ and (2.4) holds again.

From (2.2) we deduce that $\sigma_{n} \in \operatorname{dom} I_{\mathcal{K}\left(v_{n}, w_{n}\right)}=\mathcal{K}\left(v_{n}, w_{n}\right)$, i.e. $I_{\mathcal{K}\left(v_{n}, w_{n}\right)}\left(\sigma_{n}\right)=$ 0 . From the Mosco-convergence established above we obtain

$$
0 \leq I_{\mathcal{K}(v, w)}(\sigma) \leq \liminf _{n \rightarrow \infty} I_{\mathcal{K}\left(v_{n}, w_{n}\right)}\left(\sigma_{n}\right)=0 .
$$

Hence, $\sigma \in \operatorname{dom} I_{\mathcal{K}(v, w)}$. Furthermore, for any $z \in \operatorname{dom} I_{\mathcal{K}(v, w)}$ there exists a sequence $z_{n} \in \operatorname{dom} I_{\mathcal{K}\left(v_{n}, w_{n}\right)}, z_{n} \rightarrow z$ in $L^{2}(0, T ; H)$ such that $I_{\mathcal{K}\left(v_{n}, w_{n}\right)}\left(z_{n}\right) \rightarrow$ $I_{\mathcal{K}(v, w)}(z)$. The definition of the subdifferential together with (2.2) imply that

$$
\left(f_{n}, z_{n}-\sigma_{n}\right)_{L^{2}(0, T ; H)} \leq I_{\mathcal{K}\left(v_{n}, w_{n}\right)}\left(z_{n}\right)-I_{\mathcal{K}\left(v_{n}, w_{n}\right)}\left(\sigma_{n}\right)=0 .
$$


Passing to the limit in this inequality we obtain

$$
(f, z-\sigma)_{L^{2}(0, T ; H)} \leq 0=I_{\mathcal{K}(v, w)}(z)-I_{\mathcal{K}(v, w)}(\sigma) .
$$

Since $z \in \operatorname{dom} I_{\mathcal{K}(v, w)}$ is arbitrary, from this inequality it follows that

$$
f \in \partial I_{\mathcal{K}(v, w)}(\sigma)
$$

The claim of the lemma finally follows from [31, Proposition 0.3.3].

In the rest of the paper, $H$ denotes the Hilbert space $L^{2}(\Omega)$ endowed with the standard inner product $(\cdot, \cdot)_{H}$ and the associated norm $|\cdot|_{H}$, and $V$ denotes the Sobolev space $H^{1}(\Omega)$ equipped with the norm $|v|_{V}=(v, v)_{V}^{1 / 2}$, where $(v, w)_{V}=$ $(v, w)_{H}+a(v, w)$

$$
a(v, w)=\int_{\Omega} \nabla v(x) \cdot \nabla w(x) d x, \quad v, w \in V,
$$

$H^{2}(\Omega)$ is the Sobolev space $W^{2,2}(\Omega)$. Consider the linear continuous operator $\mathcal{L}$ : $V \rightarrow V^{\prime}$ defined by

$$
\langle\mathcal{L} v, w\rangle=a(v, w), \quad v, w \in V,
$$

where $V^{\prime}$ is the dual space of $V$ and $\langle\cdot, \cdot\rangle$ is the bilinear form establishing the duality between $V$ and $V^{\prime}$. Let $-\Delta_{N}: D\left(-\Delta_{N}\right) \subset H \rightarrow H$ denote the restriction of the operator $\mathcal{L}$ to the set of elements $v \in V$ such that $\mathcal{L} v \in H$. Then, $D\left(-\Delta_{N}\right)=$ $\left\{v \in H^{2}(\Omega) ; \partial v / \partial n=0\right.$ in $\left.H^{1 / 2}(\partial \Omega)\right\}$ and $-\Delta_{N} v=-\Delta v$ for all $v \in D\left(-\Delta_{N}\right)$.

Problem $(P)$ is considered under the following hypotheses:

Hypotheses (H). The following assumptions hold throughout the paper:

(H1) $\kappa>0$ is a given constant, $\lambda \in C^{2}(\mathbb{R})$ is a given function with $\lambda^{\prime}, \lambda^{\prime \prime}$ bounded on $\mathbb{R}$;

(H2) $f_{*}, f^{*} \in C^{2}\left(\mathbb{R}^{2}\right) \cap W^{2, \infty}\left(\mathbb{R}^{2}\right)$ with $0 \leq f_{*} \leq f^{*} \leq 1$ on $\mathbb{R}^{2}$, and $h(\sigma, 0, w)=0$ for $\sigma \in[0,1], w \in \mathbb{R}, g(\sigma, v, 0)=0$ for $\sigma \in[0,1], v \in \mathbb{R}$;

(H3) $F, h, g: \mathbb{R}^{3} \rightarrow \mathbb{R}$ are locally Lipschitz continuous functions;

(H4) $\sigma_{0}, v_{0}, w_{0} \in L^{\infty}(\Omega) \cap V$ with $v_{0} \geq 0, w_{0} \geq 0$ and $f_{*}\left(v_{0}, w_{0}\right) \leq \sigma_{0} \leq f^{*}\left(v_{0}, w_{0}\right)$ a.e. on $\Omega$.

We note that the bounds for $f_{*}$ and $f^{*}$ in $(H 2)$ are justified from the biological point of view. Indeed, when the prey population $v$ is zero, the vegetation $\sigma$ in our three-species model stays constant, say it is one, after rescaling. And if $v$ reaches some excessive number, then all the vegetation is devoured. So, we may assume that $\sigma=0$ in this case.

Denote by $\mathbb{R}^{+}:=[0,+\infty)$. In connection with the constraint (1.6), we assume the following:

Hypotheses (U). The multivalued mapping $U:[0, T] \times \Omega \times \mathbb{R}^{3} \rightarrow c b(\mathbb{R})$ has the following properties: 
(U1) the mapping $(t, x) \rightarrow U(t, x, \sigma, v, w), \sigma, v, w \in \mathbb{R}$, is measurable;

(U2) there exists $k \in L^{2}\left(0, T ; \mathbb{R}^{+}\right)$such that

$$
\begin{aligned}
& \operatorname{haus}_{\mathbb{R}}\left(U\left(t, x, \sigma_{1}, v_{1}, w_{1}\right)\right.\left., U\left(t, x, \sigma_{2}, v_{2}, w_{2}\right)\right) \\
& \leq k(t)\left(\left|\sigma_{1}-\sigma_{2}\right|+\left|v_{1}-v_{2}\right|+\left|w_{1}-w_{2}\right|\right)
\end{aligned}
$$

a.e. on $Q(T), \sigma_{i}, v_{i}, w_{i} \in \mathbb{R}, i=1,2$;

(U3) there exists a constant $m>0$ such that

$$
|U(t, x, \sigma, v, w)| \leq m \quad \text { a.e. on } Q(T), \sigma, v, w \in \mathbb{R} .
$$

The last set of hypotheses lists the assumptions we impose on the cost integrand:

Hypotheses (q). The function $q: \Omega_{T} \times \mathbb{R}^{3} \times \mathbb{R} \rightarrow \mathbb{R}$ is such that

(q1) the function $(t, x) \mapsto q(t, x, \sigma, v, w, u)$ is measurable;

(q2) there exist $k_{0} \in L^{2}\left(Q(T) ; \mathbb{R}^{+}\right)$such that

$$
\begin{aligned}
\mid q\left(t, x, \sigma_{1}, v_{1}, w_{1}, u_{1}\right) & -q\left(t, x, \sigma_{2}, v_{2}, w_{2}, u_{2}\right) \mid \\
& \leq k_{0}(t, x)\left(\left|\sigma_{1}-\sigma_{2}\right|+\left|v_{1}-v_{2}\right|+\left|w_{1}-w_{2}\right|+\left|u_{1}-u_{2}\right|\right)
\end{aligned}
$$

a.e. on $Q(T), \sigma_{i}, v_{i}, w_{i} \in \mathbb{R},\left|u_{i}\right| \leq m, i=1,2$;

(q3) there exist functions $k_{i} \in L^{1}\left(Q(T) ; \mathbb{R}^{+}\right), i=1,2, k_{3} \in L^{2}\left(Q(T) ; \mathbb{R}^{+}\right)$such that

$$
|q(t, x, \sigma, v, w, u)| \leq k_{1}(t, x)+k_{2}(t, x)|u|+k_{3}(t, x)(|\sigma|+|v|+|w|)
$$

a.e. on $\Omega_{T}, \sigma, v, w \in \mathbb{R},|u| \leq m$.

Next, we reformulate our problem $(P)$ in a function spaces framework. To this end, define the multivalued mapping

$\mathcal{U}(t, \sigma, v, w)=\{u \in H ; u(x) \in U(t, x, \sigma(x), v(x), w(x))$ a.e. on $\Omega\}, \quad \sigma, v, w \in H$,

the function

$$
\mathfrak{q}(t, \sigma, v, w, u)=\int_{\Omega} q(t, x, \sigma(x), v(x), w(x), u(x)) d x, \quad \sigma, v, w, u \in H,
$$

and the set

$\mathcal{K}(v, w)=\left\{\sigma \in H ; f_{*}(v(x), w(x)) \leq \sigma(x) \leq f^{*}(v(x), w(x)) \quad\right.$ a.e. on $\left.\Omega\right\}, \quad v, w \in H$,

Theorem 1.5 in [32] implies that

$$
\overline{\operatorname{co}} \mathcal{U}(t, \sigma, v, w)=\{u \in H ; u(x) \in \operatorname{co} U(t, x, \sigma(x), v(x), w(x)) \text { a.e. on } \Omega\} .
$$

Given Hypotheses $(U)$ and $(q)$ it is a routine matter to verify (cf., e.g., 7, Lemmas 3.1 and 3.2]) that the mapping $\mathcal{U}:[0, T] \times H^{3} \rightarrow c b(H)$ has the properties: 
$(\mathcal{U} 1)$ the mapping $t \mapsto \mathcal{U}(t, \sigma, v, w)$ is measurable, $\sigma, v, w \in H$;

$(\mathcal{U} 2) \operatorname{haus}_{H}\left(\mathcal{U}\left(t, \sigma_{1}, v_{1}, w_{1}\right), \mathcal{U}\left(t, \sigma_{2}, v_{2}, w_{2}\right)\right)$

$$
\leq k(t)\left(\left|\sigma_{1}-\sigma_{2}\right|_{H}+\left|v_{1}-v_{2}\right|_{H}+\left|w_{1}-w_{2}\right|_{H}\right)
$$

a.e. on $[0, T], \sigma_{i}, v_{i}, w_{i} \in H, i=1,2$, for $k \in L^{2}\left(0, T ; \mathbb{R}^{+}\right)$as above;

(U3) $|\mathcal{U}(t, \sigma, v, w)|_{H} \leq m \mu(\Omega)^{1 / 2}$ a.e. on $[0, T], \sigma, v, w \in H$, where $m>0$ is as above and $\mu(\Omega)$ is the Lebesgue measure of $\Omega$,

and the function $\mathfrak{q}:[0, T] \times H^{3} \times H \rightarrow \mathbb{R}$ has the properties:

(q1) the function $t \mapsto \mathfrak{q}(t, \sigma, v, w, u)$ is measurable, $\sigma, v, w, u \in H$;

(q2) $\left.\mid \mathfrak{q}\left(t, \sigma_{1}, v_{1}, w_{1}, u_{1}\right)-\mathfrak{q}\left(t, \sigma_{2}, v_{2}, w_{2}, u_{2}\right)\right)$

$$
\leq k_{0}^{*}(t)\left(\left|v_{1}-v_{2}\right|_{H}+\left|w_{1}-w_{2}\right|_{H}+\left|\sigma_{1}-\sigma_{2}\right|_{H}+\left|u_{1}-u_{2}\right|_{H}\right)
$$

a.e. on $[0, T], \sigma_{i}, v_{i}, w_{i}, u_{i} \in H, i=1,2$;

(q3) $|\mathfrak{q}(t, \sigma, v, w, u)| \leq k_{1}^{*}(t)+k_{2}^{*}(t) m+k_{3}^{*}(t)\left(|\sigma|_{H}+|v|_{H}+|w|_{H}\right)$

a.e. on $[0, T], \sigma, v, w, u \in H$.

Here, $k_{i}^{*}(t)=\left|k_{i}(t)\right|_{L^{1}(\Omega)}, i=1,2, k_{j}^{*}(t)=\left|k_{j}(t)\right|_{H}, j=0,3$.

Let $\partial I_{\mathcal{K}(v, w)}(\sigma)$ be the subdifferential of the indicator function of $\mathcal{K}(v, w)$ at a point $\sigma \in H$. Now we are in a position to define solutions for our control problems.

Definition 2.1. A quadruple $\{\sigma, v, w, u\}$ is called a solution of control system (1.1)(1.6) if

(i) $\sigma, v, w \in W^{1,2}(0, T ; H) \cap L^{\infty}(0, T ; V) \cap L^{2}\left(0, T ; H^{2}(\Omega)\right) ;$

(ii) $u \in L^{2}(0, T ; H)$;

(iii) $\sigma^{\prime}-(\lambda(v))^{\prime}-\kappa \Delta_{N} \sigma+\partial I_{\mathcal{K}(v, w)}(\sigma) \ni F(\sigma, v, w) u \quad$ in $H$ a.e. on $[0, T]$;

(iv) $v^{\prime}-\Delta_{N} v=h(\sigma, v, w)$ in $H$ a.e. on $[0, T]$;

(v) $w^{\prime}-\Delta_{N} w=g(\sigma, v, w)$ in $H$ a.e. on $[0, T]$;

(vi) $\sigma(0)=\sigma_{0}, v(0)=v_{0}, w(0)=w_{0} \quad$ in $H$;

(vii) $u(t) \in \mathcal{U}(t, v(t), w(t), \sigma(t)) \quad$ in $H$ a.e. on $[0, T]$,

where the prime denotes derivative with respect to $t$.

A solution of control system (1.1)-(1.5), (1.7) is defined similarly replacing the last inclusion with

$$
u(t) \in \overline{\mathrm{co}} \mathcal{U}(t, \sigma(t), v(t), w(t)) \quad \text { in } H \text { for a.e. } t \in[0, T]
$$


The sets of all solutions to control systems (1.1)-(1.6) and (1.1)-(1.5), (1.7) in the sense of Definition 2.1 we denote by $\mathcal{R}_{\mathcal{U}}$ and $\mathcal{R}_{\overline{\mathrm{co}} \mathcal{U}}$, respectively.

Defining the function $\mathfrak{q}_{\mathcal{U}}:[0, T] \times H^{3} \times H \rightarrow \mathbb{R} \cup\{+\infty\}$ by the rule

$$
\mathfrak{q}_{\mathcal{u}}(t, \sigma, v, w, u)= \begin{cases}\mathfrak{q}(t, \sigma, v, w, u) & \text { if } u \in \mathcal{U}(t, \sigma, v, w) \\ +\infty & \text { otherwise }\end{cases}
$$

and denoting by $\mathfrak{q}_{\mathcal{u}}^{* *}(t, \sigma, v, w, u)$ the bipolar of the function $u \mapsto \mathfrak{q}_{\mathcal{u}}(t, \sigma, v, w, u)$, one can easily see that

$$
\mathfrak{q}_{\mathcal{U}}^{* *}(t, \sigma, v, w, u)=\int_{\Omega} q_{U}^{* *}(t, x, \sigma(x), v(x), w(x), u(x)) d x .
$$

So, our optimal control Problems $(P)$ and $(R P)$ can now be reformulated in the form:

(P) minimize $J(\sigma, v, w, u)=\int_{0}^{T} \mathfrak{q}(t, \sigma(t), v(t), w(t), u(t)) d t \quad$ over $\mathcal{R}$,

$(\mathrm{RP})$ minimize $\quad J_{U}^{* *}(\sigma, v, w, u)=\int_{0}^{T} \mathfrak{q}_{\mathcal{U}}^{* *}(t, \sigma(t), v(t), w(t), u(t)) d t \quad$ over $\quad \mathcal{R}_{\overline{\mathrm{co}} \mathcal{U}}$.

Given Hypotheses $(H),(U)$, and $(q)$, the main purpose of this work is to prove the following results.

Theorem 2.1. For any $\left(\sigma_{*}, v_{*}, w_{*}, u_{*}\right) \in \mathcal{R}_{\overline{\mathrm{co}} \mathcal{U}}$ there exists a sequence $\left(\sigma_{n}, v_{n}, w_{n}, u_{n}\right)$ $\in \mathcal{R}_{\mathcal{U}}, n \geq 1$, such that

$$
\begin{aligned}
\left(\sigma_{n}, v_{n}, w_{n}\right) & \rightarrow\left(\sigma_{*}, v_{*}, w_{*}\right) \quad \text { strongly in } C\left([0, T], H^{3}\right), \\
u_{n} & \rightarrow u_{*} \quad \text { weakly in } L^{2}([0, T], H) .
\end{aligned}
$$

Moreover,

$$
\begin{aligned}
\lim _{n \rightarrow \infty} \sup _{0 \leq s \leq t \leq T} \mid \int_{s}^{t} & \left(\mathfrak{q}_{\mathcal{U}}^{* *}\left(\tau, \sigma_{*}(\tau), v_{*}(\tau), w_{*}(\tau), u_{*}(\tau)\right)\right. \\
& \left.\quad-\mathfrak{q}\left(\tau, \sigma_{n}(\tau), v_{n}(\tau), w_{n}(\tau), u_{n}(\tau)\right)\right) d \tau \mid=0
\end{aligned}
$$

Theorem 2.2. Problem (RP) has an optimal solution and

$$
\min _{(\sigma, v, w, u) \in \mathcal{R}_{\overline{\mathrm{co}} \mathcal{U}}} J_{U}^{* *}(\sigma, v, w, u)=\inf _{(\sigma, v, w, u) \in \mathcal{R}_{\mathcal{U}}} J(\sigma, v, w, u) .
$$

Moreover, for any solution $\left(\sigma_{*}, v_{*}, w_{*}, u_{*}\right)$ of $(\mathrm{RP})$ there exists a minimizing sequence $\left(\sigma_{n}, v_{n}, w_{n}, u_{n}\right) \in \mathcal{R}_{\mathcal{U}}, n \geq 1$, for problem $(\mathrm{P})$ such that (2.5)-(2.7) hold. 


\section{Properties of the control-to-state solution oper- ator}

In this section, we define the control-to-state solution operator for our control systems and explore some of its properties which are crucial for establishing our main results in the next section. To this aim, first we let

$$
S_{m}:=\left\{u \in L^{2}(0, T ; H) ;|u(t, x)| \leq m \quad \text { a.e. on } Q(T)\right\} .
$$

Due to convexity, the bound for the control functions of Problem $(P)$ given in Hypothesis $(U 3)$ obviously extends to the control functions of the convexified problem $(R P)$. In particular, the controls of both problems belong to the set $S_{m}$. Accordingly, let $\mathcal{T}: S_{m} \rightarrow C([0, T] ; H \times H \times H)$ be the operator which with each $u \in S_{m}$ associates the unique solution of system (1.1)-(1.5):

$$
\left(\sigma_{u}, v_{u}, w_{u}\right)=\mathcal{T}(u)
$$

The existence and uniqueness of such a solution as well as uniform a priori estimates for all possible solutions independent of the control $u$ are provided by the following theorem.

Theorem 3.1. For any fixed $u \in S_{m}$ system (1.1) (1.5) has a unique solution. Moreover, for any solution $(v, w, \sigma)$ of (1.1) -(1.5) with $u \in S_{m}$ the following estimates

$$
\begin{gathered}
0 \leq \sigma, v, w \leq M_{0} \quad \text { a.e. on } Q(T), \\
\left|\sigma^{\prime}\right|_{L^{2}(0, T ; H)}+\left|v^{\prime}\right|_{L^{2}(0, T ; H)}+\left|w^{\prime}\right|_{L^{2}(0, T ; H)} \\
+|\Delta \sigma|_{L^{2}(0, T ; H)}+|\Delta v|_{L^{2}(0, T ; H)}+|\Delta w|_{L^{2}(0, T ; H)} \\
+|\nabla \sigma|_{L^{\infty}(0, T ; H)}+|\nabla v|_{L^{\infty}(0, T ; H)}+|\nabla w|_{L^{\infty}(0, T ; H)} \leq M_{0}
\end{gathered}
$$

hold for a constant $M_{0}>0$ independent of $u$.

Proof. The existence of a unique solution to (1.1)-(1.5) with a fixed $u \in S_{m}$ and the estimate (3.2) can be proved following the pattern of 23. Theorems 3.1,3.2, and 3.10].

By virtue of the bound (3.2), we may now assume (cutting off outside the set where $\sigma, v$, and $w$ are bounded, if necessary) that the functions $F, h, g$ are all bounded (with a common bound $M>0$ ) and globally Lipschitz continuous (with a common Lipschitz constant $L>1$ ).

Below, we recap a part of the reasoning of [23] which will allow us to establish the uniform energy estimates (3.3). To this end, given $\mu>0$ we introduce the following approximate system:

$$
\begin{gathered}
\sigma_{\mu}^{\prime}-\left(\lambda\left(v_{\mu}\right)\right)^{\prime}-\kappa \Delta_{N} \sigma_{\mu}+\partial I_{\mathcal{K}\left(v_{\mu}, w_{\mu}\right)}^{\mu}\left(\sigma_{\mu}\right)=F\left(\sigma_{\mu}, v_{\mu}, w_{\mu}\right) u \text { in } H \text { a.e. on }[0, T] \\
v_{\mu}^{\prime}-\Delta_{N} v_{\mu}=h\left(\sigma_{\mu}, v_{\mu}, w_{\mu}\right) \quad \text { in } H \text { a.e. on }[0, T]
\end{gathered}
$$




$$
w_{\mu}^{\prime}-\Delta_{N} w_{\mu}=g\left(\sigma, v_{\mu}, w_{\mu}\right) \quad \text { in } H \text { a.e. on }[0, T] .
$$

Here, for $\mu>0$ and $\sigma, v, w \in \mathbb{R}$ the function $\partial I_{\mathcal{K}(v, w)}^{\mu}(\sigma)$ is the Yosida regularization of the subdifferential $\partial I_{\mathcal{K}(v, w)}(\sigma)$.

By [23, for any $\mu>0$ there exists a unique triplet $\left(\sigma_{\mu}, v_{\mu}, w_{\mu}\right)$ solving the approximate system above and satisfying the initial conditions $\sigma_{\mu}(0)=\sigma_{0}, v_{\mu}(0)=$ $v_{0}, w_{\mu}(0)=w_{0}$. Arguing similarly to [23, 3.3.2. Proof of Theorem 3.6] we obtain the following counterparts of the inequalities $(21),(22),(23),(32)$ of this reference for such triplets $\left(\sigma_{\mu}, v_{\mu}, w_{\mu}\right), \mu>0$ :

$$
\begin{gathered}
\left|v_{\mu}^{\prime}\right|_{H}^{2}+\left|w_{\mu}^{\prime}\right|_{H}^{2}+\frac{d}{d t}\left|\nabla v_{\mu}\right|_{H}^{2}+\frac{d}{d t}\left|\nabla w_{\mu}\right|_{H}^{2} \leq C_{1} \\
\frac{d}{d t}\left|\nabla v_{\mu}\right|_{H}^{2}+\frac{d}{d t}\left|\nabla w_{\mu}\right|_{H}^{2}+\left|\Delta v_{\mu}\right|_{H}^{2}+\left|\Delta w_{\mu}\right|_{H}^{2} \leq C_{1} \\
\left|\sigma_{\mu}^{\prime}\right|_{H}^{2}+\kappa \frac{d}{d t}\left|\nabla \sigma_{\mu}\right|_{H}^{2}+2 \frac{d}{d t} I_{\mathcal{K}\left(v_{\mu}, w_{\mu}\right)}^{\mu}(\sigma) \leq C_{2}\left(\left|v_{\mu}^{\prime}\right|_{H}^{2}+\left|w_{\mu}^{\prime}\right|_{H}^{2}+\kappa^{2}\left|\Delta \sigma_{\mu}\right|_{H}^{2}+1\right) \\
\frac{d}{d t}\left\{I_{v_{\mu}, w_{\mu}}^{\mu}\left(\sigma_{\mu}\right)+\frac{1}{2}\left|\nabla \sigma_{\mu}\right|_{H}^{2}-\left(\lambda^{\prime}\left(v_{\mu}\right) \nabla v_{\mu}, \nabla \sigma_{\mu}\right)_{H}\right\} \\
+\kappa\left|\Delta \sigma_{\mu}\right|_{H}^{2}+\frac{1-\kappa}{4}\left|\partial I_{\mathcal{K}\left(v_{\mu}, w_{\mu}\right)}^{\mu}\left(\sigma_{\mu}\right)\right|_{H}^{2} \\
\leq C_{3}\left(1+\left|\nabla \sigma_{\mu}\right|_{H}^{2}+\left|\nabla v_{\mu}\right|_{H}^{2}+\left|\nabla w_{\mu}\right|_{H}^{2}+\left|\sigma_{\mu}^{\prime}\right|_{H}^{2}+\left|v_{\mu}^{\prime}\right|_{H}^{2}+\left|w_{\mu}^{\prime}\right|_{H}^{2}\right. \\
\left.+(1+\kappa)\left(1+\left|\Delta v_{\mu}\right|_{H}^{2}+\left|\Delta w_{\mu}\right|_{H}^{2}\right)\right)
\end{gathered}
$$

a.e. on $(0, T)$, where $C_{1}, C_{2}, C_{3}$ are some positive constants depending on $\left|\lambda^{\prime}\right|_{L^{\infty}(\mathbb{R})}$, $\left|\lambda^{\prime \prime}\right|_{L^{\infty}(\mathbb{R})},\left|f_{*}\right|_{W^{2, \infty}\left(\mathbb{R}^{2}\right)},\left|f_{*}\right|_{W^{2, \infty}\left(\mathbb{R}^{2}\right)}, L, M_{0}, M, m$, and $\mu(\Omega)$, but independent of $\mu$. Next, calculating (3.7) $+\varepsilon_{1} \times(3.8)+\varepsilon_{2} \times(3.9)+\varepsilon_{3} \times(3.10)$ with positive numbers $\varepsilon_{1}, \varepsilon_{2}, \varepsilon_{3}$ to be specified later we obtain

$$
\begin{aligned}
& \left(\varepsilon_{2} C_{2}-\varepsilon_{3} C_{3}\right)\left|\sigma_{\mu}^{\prime}\right|_{H}^{2}+\left(1-\varepsilon_{2} C_{2}-\varepsilon_{3} C_{3}\right)\left|v_{\mu}^{\prime}\right|_{H}^{2}+\left(1-\varepsilon_{2} C_{2}-\varepsilon_{3} C_{3}\right)\left|w_{\mu}^{\prime}\right|_{H}^{2} \\
& +\kappa\left(\varepsilon_{3}-\varepsilon_{2} C_{2} \kappa\right)\left|\Delta \sigma_{\mu}\right|_{H}^{2}+\left(\varepsilon_{1}-\varepsilon_{3} C_{3}(1+\kappa)\right)\left|\Delta v_{\mu}\right|_{H}^{2}+\left(\varepsilon_{1}-\varepsilon_{3} C_{3}(1+\kappa)\right)\left|\Delta w_{\mu}\right|_{H}^{2} \\
& +\varepsilon_{3} \frac{1-\kappa}{4}\left|\partial I_{\mathcal{K}\left(v_{\mu}, w_{\mu}\right)}^{\mu}\left(\sigma_{\mu}\right)\right|_{H}^{2}+\frac{d}{d t}\left\{\left(2 \varepsilon_{2}+\varepsilon_{3}\right) I_{\mathcal{K}\left(v_{\mu}, w_{\mu}\right)}^{\mu}\left(\sigma_{\mu}\right)-\varepsilon_{3}\left(\lambda^{\prime}\left(v_{\mu}\right) \nabla v_{\mu}, \nabla \sigma_{\mu}\right)_{H}\right. \\
& \left.+\left(\varepsilon_{2} \kappa+\frac{\varepsilon_{3}}{2}\right)\left|\nabla \sigma_{\mu}\right|_{H}^{2}+\left(1+\varepsilon_{1}\right)\left|\nabla v_{\mu}\right|_{H}^{2}+\left(1+\varepsilon_{1}\right)\left|\nabla w_{\mu}\right|_{H}^{2}\right\} \\
& \leq C_{4}+\varepsilon_{3} C_{3}\left(2+\kappa+\left|\nabla \sigma_{\mu}\right|_{H}^{2}+\left|\nabla v_{\mu}\right|_{H}^{2}+\left|\nabla w_{\mu}\right|_{H}^{2}\right),
\end{aligned}
$$

where $C_{4}=C_{1}+\varepsilon_{1} C_{1}+\varepsilon_{2} C_{2}$. Integrating this inequality from 0 to $t \in(0, T]$ and then estimating the term $-\varepsilon_{3}\left(\lambda^{\prime}\left(v_{\mu}\right) \nabla v_{\mu}, \nabla \sigma_{\mu}\right)_{H}$ in the resulting inequality as follows

$$
-\varepsilon_{3}\left(\lambda^{\prime}\left(v_{\mu}\right) \nabla v_{\mu}, \nabla \sigma_{\mu}\right)_{H} \geq-\frac{1}{4} \varepsilon_{3}^{2}\left|\lambda^{\prime}\right|_{L^{\infty}(\mathbb{R})}^{2}\left|\nabla \sigma_{\mu}\right|_{H}^{2}-\left|\nabla v_{\mu}\right|_{H}^{2}
$$


we infer that

$$
\begin{aligned}
&\left(\varepsilon_{2} C_{2}-\varepsilon_{3} C_{3}\right)\left|\sigma_{\mu}^{\prime}\right|_{L^{2}(0, t ; H)}^{2}+\left(1-\varepsilon_{2} C_{2}-\varepsilon_{3} C_{3}\right)\left|v_{\mu}^{\prime}\right|_{L^{2}(0, t ; H)}^{2} \\
&+\left(1-\varepsilon_{2} C_{2}-\varepsilon_{3} C_{3}\right)\left|w_{\mu}^{\prime}\right|_{L^{2}(0, t ; H)}^{2} \\
&+\kappa\left(\varepsilon_{3}-\varepsilon_{2} C_{2} \kappa\right)\left|\Delta \sigma_{\mu}\right|_{L^{2}(0, t ; H)}^{2}+\left(\varepsilon_{1}-\varepsilon_{3} C_{3}(1+\kappa)\right)\left|\Delta v_{\mu}\right|_{L^{2}(0, t ; H)}^{2} \\
&+\left(\varepsilon_{1}-\varepsilon_{3} C_{3}(1+\kappa)\right)\left|\Delta w_{\mu}\right|_{L^{2}(0, t ; H)}^{2} \\
&+\varepsilon_{3} \frac{1-\kappa}{4}\left|\partial I_{\mathcal{K}\left(v_{\mu}, w_{\mu}\right)}^{\mu}\left(\sigma_{\mu}\right)\right|_{L^{2}(0, t ; H)}^{2}+\left(2 \varepsilon_{2}+\varepsilon_{3}\right) I_{\mathcal{K}\left(v_{\mu}, w_{\mu}\right)}^{\mu}\left(\sigma_{\mu}\right) \\
&+\left(\varepsilon_{2} \kappa+\frac{\varepsilon_{3}}{2}-\frac{1}{4} \varepsilon_{3}^{2}\left|\lambda^{\prime}\right|_{L^{\infty}(\mathbb{R})}^{2}\right)\left|\nabla \sigma_{\mu}\right|_{H}^{2}+\varepsilon_{1}\left|\nabla v_{\mu}\right|_{H}^{2}+\left(1+\varepsilon_{1}\right)\left|\nabla w_{\mu}\right|_{H}^{2} \\
& \leq C_{5}+\varepsilon_{3} C_{3} \int_{0}^{t}\left\{\left|\nabla \sigma_{\mu}(\tau)\right|_{H}^{2}+\left|\nabla v_{\mu}(\tau)\right|_{H}^{2}+\left|\nabla w_{\mu}(\tau)\right|_{H}^{2}\right\} d \tau,
\end{aligned}
$$

where $C_{5}$ is a positive constant which depends on $\left|\sigma_{0}\right|_{V},\left|v_{0}\right|_{V},\left|w_{0}\right|_{V}$, but is independent of $\mu$. Now, all the coefficients in (3.11) will be positive provided we take $\varepsilon_{1}=\min \left\{\min \left\{1, \frac{1}{2 C_{2}}\right\}, \frac{4 C_{3}}{\left|\lambda^{\prime}\right|_{L}^{2} \infty_{(\mathbb{R})}}\right\}, \varepsilon_{2}=\frac{1}{4 C_{2}}, \varepsilon_{3}=\min \left\{\frac{1}{4 C_{3}} \min \left\{1, \frac{1}{2 C_{2}}\right\}, \frac{1}{\left|\lambda^{\prime}\right|_{L}^{2} \infty_{(\mathbb{R})}}\right\}$ and choose $\kappa \in\left(0, \kappa_{0}\right)$ with $\kappa_{0}=\min \left\{\frac{1}{2}, \frac{1}{2 C_{3}} \min \left\{1, \frac{1}{2 C_{2}}\right\}, \frac{2}{\left|\lambda^{\prime}\right|_{L}^{2} \infty_{(\mathbb{R})}}\right\}$. Therefore, invoking Gronwall's inequality from (3.11) we obtain the following uniform energy estimates for the triplets $\left(\sigma_{\mu}, v_{\mu}, w_{\mu}\right), \mu>0$, solving the approximate problem (3.4) $-(3.6)$ :

$$
\begin{aligned}
& \left|\sigma_{\mu}^{\prime}\right|_{L^{2}(0, T ; H)}+\left|v_{\mu}^{\prime}\right|_{L^{2}(0, T ; H)}+\left|w_{\mu}^{\prime}\right|_{L^{2}(0, T ; H)} \\
+ & \left|\Delta \sigma_{\mu}\right|_{L^{2}(0, T ; H)}+\left|\Delta v_{\mu}\right|_{L^{2}(0, T ; H)}+\left|\Delta w_{\mu}\right|_{L^{2}(0, T ; H)} \\
+ & \left|\nabla \sigma_{\mu}\right|_{L^{\infty}(0, T ; H)}+\left|\nabla v_{\mu}\right|_{L^{\infty}(0, T ; H)}+\left|\nabla w_{\mu}\right|_{L^{\infty}(0, T ; H)} \\
+ & \left|\partial I_{\mathcal{K}\left(v_{\mu}, w_{\mu}\right)}^{\mu}\left(\sigma_{\mu}\right)\right|_{L^{2}(0, T ; H)}+\left|I_{\mathcal{K}\left(v_{\mu}, w_{\mu}\right)}^{\mu}\left(\sigma_{\mu}\right)\right|_{L^{\infty}(0, T)} \leq M_{0},
\end{aligned}
$$

By the weak and weak-star compactness results, this bound allows us to conclude that there exists a null sequence $\mu_{n}, n \geq 1$, and functions $\sigma, v, w \in W^{1,2}(0, T ; H) \cap$ $L^{\infty}(0, T ; V) \cap L^{2}\left(0, T ; H^{2}(\Omega)\right)$ such that

$$
\sigma_{n}:=\sigma_{\mu_{n}} \rightarrow \sigma, \quad v_{n}:=v_{\mu_{n}} \rightarrow v, \quad w_{n}:=w_{\mu_{n}} \rightarrow w
$$

weakly in $W^{1,2}(0, T ; H) \cap L^{2}\left(0, T ; H^{2}(\Omega)\right)$ and weakly-star in $L^{\infty}(0, T ; V)$, and, thus, strongly in $C([0, T] ; H)$.

With the convergences (3.13) at hand, we can now pass to the limit in Eqs. (3.5), (3.6) to infer that the triplet $(\sigma, v, w)$ satisfies Eqs. (iv), $(v)$ of Definition 2.1. Furthermore, denoting $f_{n}:=-\sigma_{n}^{\prime}+\left(\lambda\left(v_{n}\right)\right)^{\prime}+\kappa \Delta_{N} \sigma_{n}+F\left(\sigma_{n}, v_{n}, w_{n}\right) u$ we see that

$$
f_{n} \rightarrow f:=-\sigma^{\prime}+(\lambda(v))^{\prime}+\kappa \Delta_{N} \sigma+F(\sigma, v, w) u
$$

weakly in $L^{2}(0, T ; H)$. Since [31, Proposition 0.3.5]

$$
\partial I_{\mathcal{K}\left(v_{n}, w_{n}\right)}^{\mu_{n}}\left(\sigma_{n}\right) \in \partial I_{\mathcal{K}\left(v_{n}, w_{n}\right)}\left(J_{\mathcal{K}\left(v_{n}, w_{n}\right)}\left(\sigma_{n}\right)\right),
$$


where $J_{\mathcal{K}\left(v_{n}, w_{n}\right)}\left(\sigma_{n}\right)$ is the projection of $\sigma_{n}$ onto the set $\mathcal{K}\left(v_{n}, w_{n}\right)$, we also have

$$
f_{n} \in \partial I_{\mathcal{K}\left(v_{n}, w_{n}\right)}\left(J_{\mathcal{K}\left(v_{n}, w_{n}\right)}\left(\sigma_{n}\right)\right) .
$$

On the other hand, the boundedness of $I_{\mathcal{K}\left(v_{n}, w_{n}\right)}^{\mu_{n}}\left(\sigma_{n}\right)$ implies that

$$
J_{\mathcal{K}\left(v_{n}, w_{n}\right)}\left(\sigma_{n}\right) \rightarrow \sigma
$$

strongly in $C([0, T] ; H)$. In view of (3.13)-(3.16), the application of Lemma 2.1 yields

$$
f \in \partial I_{\mathcal{K}(v, w)}(\sigma),
$$

so that the triplet $(\sigma, v, w)$ satisfies Eqs. (iii) of Definition 2.1 as well. We note that inequality (2.1) in this case follows from the Lipschitzness of $f_{*}$ and $f^{*}$. Therefore, we conclude that $(\sigma, v, w)$ is a solution of system (1.1) (1.5) with a fixed $u \in S_{m}$. The estimate (3.3) now follows from (3.12) and (3.13).

Theorem 3.2. The operator $\mathcal{T}: S_{m} \rightarrow C([0, T] ; H \times H \times H)$ is weak-strong continuous.

Proof. We note that the set $S_{m}$ when endowed with the weak topology of the space $L^{2}(0, T ; H)$ is metrizable. Consequently, to prove the theorem it is enough to show the sequential continuity of the operator $\mathcal{T}$. Let, then, $u_{n}, n \geq 1$, be an arbitrary sequence from $S_{m}$ weakly converging to some $u \in S_{m}$. Denote by $\left(\sigma_{n}, v_{n}, w_{n}\right):=\left(\sigma\left(u_{n}\right), v\left(u_{n}\right), w\left(u_{n}\right)\right), n \geq 1$, the sequences of solutions to system (1.1)-1.5) corresponding to the controls $u_{n}, n \geq 1$. Similarly as in the proof of Theorem 3.1, by the weak and weak-star compactness results, the uniform estimates (3.2) and (3.3) imply that there exists a subsequence $\left(\sigma_{k}, v_{k}, w_{k}\right):=$ $\left(\sigma_{n_{k}}, v_{n_{k}}, w_{n_{k}}\right), k \geq 1$, of the sequence $\left(\sigma_{n}, v_{n}, w_{n}\right), n \geq 1$, and some functions $\sigma, v, w \in W^{1,2}(0, T ; H) \cap L^{\infty}(0, T ; V) \cap L^{2}\left(0, T ; H^{2}(\Omega)\right)$ such that

$$
\sigma_{k} \rightarrow \sigma, \quad v_{k} \rightarrow v, \quad w_{k} \rightarrow w
$$

weakly in $W^{1,2}(0, T ; H) \cap L^{2}\left(0, T ; H^{2}(\Omega)\right)$ and weakly-star in $L^{\infty}(0, T ; V)$, and, thus, strongly in $C([0, T] ; H)$.

From the Lipschitz continuity of the functions $F, g, h$ and (3.17) we deduce that

$$
\begin{aligned}
-\sigma_{k}^{\prime}+\left(\lambda\left(v_{k}\right)\right)^{\prime}+\kappa \Delta_{N} \sigma_{k} & +F\left(\sigma_{k}, v_{k}, w_{k}\right) u_{k} \\
& \rightarrow-\sigma^{\prime}+(\lambda(v))^{\prime}+\kappa \Delta_{N} \sigma+F(\sigma, v, w) u
\end{aligned}
$$

weakly in $L^{2}([0, T] ; H)$ and

$$
h\left(\sigma_{k}, v_{k}, w_{k}\right) \rightarrow h(\sigma, v, w), \quad g\left(\sigma_{k}, v_{k}, w_{k}\right) \rightarrow g(\sigma, v, w)
$$

strongly in $C([0, T] ; H)$. The claim of the theorem now follows from the convergences (3.17)-3.19) and Lemma 2.1. 
Theorem 3.3. [26, Theorem 3.2] Let $u_{i} \in S_{m}$ and $\left\{\sigma_{i}, v_{i}, w_{i}\right\}=\mathcal{T}\left(u_{i}\right), i=1,2$. Then,

$$
\begin{aligned}
\left|\sigma_{1}(t)-\sigma_{2}(t)\right|_{H}^{2}+\left|v_{1}(t)-v_{2}(t)\right|_{H}^{2} & +\left|w_{1}(t)-w_{2}(t)\right|_{H}^{2} \\
& \leq C_{m} \int_{0}^{t}\left|u_{1}(\tau)-u_{2}(\tau)\right|_{H}^{2} d \tau
\end{aligned}
$$

$t \in[0, T]$, where $C_{m}>0$ is a positive constant depending only on $m$.

\section{Proofs of the main results}

In this section, we prove Theorems 2.1 and 2.2. To this end, first, on the basis of the control constraint multivalued mapping $\mathcal{U}$ and the cost integrand $\mathfrak{q}$ we construct an auxiliary multivalued mapping $\mathcal{U}_{\mathfrak{q}}:[0, T] \times H^{3} \rightarrow H \times \mathbb{R}$ as follows

$$
\mathcal{U}_{\mathfrak{q}}(t, \sigma, v, w)=\{(u, \mathfrak{q}(t, \sigma, v, w, u)) \in H \times \mathbb{R} ; u \in \mathcal{U}(t, \sigma, v, w)\},
$$

where the Banach space $H \times \mathbb{R}$ is equipped with the norm

$$
|(u, \lambda)|_{H \times \mathbb{R}}:=\max \left\{|u|_{H},|\lambda|\right\}, \quad u \in H, \lambda \in \mathbb{R} .
$$

Given the properties $(\mathcal{U} \mathbf{1})-(\mathcal{U} \mathbf{3})$ and $(\mathfrak{q} \mathbf{1})-(\mathfrak{q} \mathbf{3})$ we can easily show that the mapping $\mathcal{U}_{\mathfrak{q}}$ is measurable in $t$, continuous in the Hausdorff metric on the space $\operatorname{cb}(H \times \mathbb{R})$ in $(\sigma, v, w)$ a.e. on $[0, T]$ and

$$
\begin{aligned}
\mid \mathcal{U}_{\mathfrak{q}}(t, \sigma, v, w) & \left.\right|_{H \times \mathbb{R}} \\
& \leq m \mu(\Omega)^{1 / 2}+k_{1}^{*}(t)+k_{2}^{*}(t) m+k_{3}^{*}(t)\left(|\sigma|_{H}+|v|_{H}+|w|_{H}\right) .
\end{aligned}
$$

Moreover, we have (cf. [33, Lemma 2.2])

$\mathfrak{q}_{\mathcal{U}}^{* *}(t, \sigma, v, w, u)= \begin{cases}\min \left\{\lambda \in \mathbb{R} ;(u, \lambda) \in \overline{\operatorname{co}} \mathcal{U}_{\mathfrak{q}}(t, \sigma, v, w)\right\} & \text { if } u \in \overline{\operatorname{co}} \mathcal{U}(t, \sigma, v, w), \\ +\infty & \text { otherwise }\end{cases}$

and for any $\varepsilon>0$, there is a closed set $T_{\varepsilon} \subset[0, T], \mu\left([0, T] \backslash T_{\varepsilon}\right) \leq \varepsilon$, such that $\mathfrak{q}_{\mathcal{U}}^{* *}$ restricted to $T_{\varepsilon} \times H^{3} \times H$ is lower semicontinuous. This implies, in particular, that given an arbitrary $\left(\sigma_{*}, v_{*}, w_{*}, u_{*}\right) \in \mathcal{R}_{\overline{\mathrm{co}} \mathcal{U}}$, the function $t \rightarrow \mathfrak{q}_{\mathcal{U}}^{* *}\left(t, \sigma_{*}(t), v_{*}(t), w_{*}(t), u_{*}(t)\right)$ is measurable and

$$
\left(u_{*}(t), \mathfrak{q}_{\mathcal{U}}^{* *}\left(t, \sigma_{*}(t), v_{*}(t), w_{*}(t), u_{*}(t)\right)\right) \in \overline{\mathrm{co}} \mathcal{U}_{\mathfrak{q}}\left(t, \sigma_{*}(t), v_{*}(t), w_{*}(t)\right)
$$

a.e. on $[0, T]$. Since the bound in (4.3) evidently extends to $\overline{c o} \mathcal{U}_{\mathfrak{q}}$, invoking [34, Corollary 1.1], from the last inclusion we obtain the existence of a measurable function $\gamma_{n}(t) \in \mathcal{U}\left(t, \sigma_{*}(t), v_{*}(t), w_{*}(t)\right)$ which, in view of (4.2), satisfies

$$
\sup _{0 \leq s \leq t \leq T}\left|\int_{s}^{t}\left(u_{*}(\tau)-\gamma_{n}(\tau)\right) d \tau\right|_{H} \leq \frac{1}{n}
$$


and

$$
\begin{aligned}
\sup _{0 \leq s \leq t \leq T} \mid \int_{s}^{t} & \left(\mathfrak{q}_{\mathcal{U}}^{* *}\left(\tau, \sigma_{*}(t), v_{*}(\tau), w_{*}(\tau), u_{*}(\tau)\right)\right. \\
& \left.-\mathfrak{q}\left(\tau, \sigma_{*}(t), v_{*}(\tau), w_{*}(\tau), \gamma_{n}(\tau)\right)\right) d \tau \mid \leq \frac{1}{n}
\end{aligned}
$$

From Theorem 3.2 it follows that the set

$$
\mathcal{R}:=\left\{(\sigma, v, w) \in C\left([0, T] ; H^{3}\right) ;(\sigma, v, w)=\mathcal{T}(u), u \in S_{m}\right\}
$$

is compact in $C\left([0, T] ; H^{3}\right)$. Fix $n \geq 1$. The property $(\mathcal{U} 2)$ implies that for any $(\sigma, v, w) \in H^{3}$ and a.e. $t \in[0, T]$ there exists $\gamma \in \mathcal{U}(t, \sigma, v, w)$ such that

$$
\begin{aligned}
\left|\gamma_{n}(t)-\gamma\right|_{H}^{2} & <\frac{2}{n^{2}}+2 d_{H}^{2}\left(\gamma_{n}(t), \mathcal{U}(t, \sigma, v, w)\right) \\
& <\frac{2}{n^{2}}+12 k^{2}(t)\left(\left|\sigma_{*}(t)-\sigma\right|_{H}^{2}+\left|v_{*}(t)-v\right|_{H}^{2}+\left|w_{*}(t)-w\right|_{H}^{2}\right) .
\end{aligned}
$$

Define the multivalued mapping

$$
\begin{aligned}
\mathcal{U}_{n}(t, \sigma, v, w) & :=\left\{\gamma \in \mathcal{U}(t, \sigma, v, w) ;\left|\gamma_{n}(t)-\gamma\right|_{H}^{2}\right. \\
& \left.\leq \frac{2}{n^{2}}+12 k^{2}(t)\left(\left|\sigma_{*}(t)-\sigma\right|_{H}^{2}+\left|v_{*}(t)-v\right|_{H}^{2}+\left|w_{*}(t)-w\right|_{H}^{2}\right)\right\}
\end{aligned}
$$

and the associated Nemytskii multivalued operator $\Gamma_{n}: \mathcal{R} \rightarrow L^{2}(0, T ; H)$ :

$$
\Gamma_{n}(\sigma, v, w):=\left\{u \in L^{2}(0, T ; H) ; u(t) \in \mathcal{U}_{n}(t, \sigma(t), v(t), w(t)) \text { for a.e. } t \in[0, T]\right\},
$$

which is lower semicontinuous with nonempty closed decomposable values. According to [35, Theorem 3.1] there exists a continuous mapping $\alpha_{n}: \mathcal{R} \rightarrow L^{1}(0, T ; H)$ such that

$$
\alpha_{n}(\sigma, v, w) \in \Gamma_{n}(\sigma, v, w),(\sigma, v, w) \in \mathcal{R} .
$$

In view of the property $(\mathcal{U} 3)$ and the definition of $\mathcal{U}_{n}$, from this inclusion we deduce that $\alpha_{n}$ is continuous from $\mathcal{R}$ to $L^{2}(0, T ; H)$ as well and $\alpha_{n}(\sigma, v, w) \in S_{m},(\sigma, v, w) \in$ $\mathcal{R}$.

Consider now the superposition of $\mathcal{T}$ and $\alpha_{n}$. By virtue of Theorem 3.2 it follows that this superposition $\alpha_{n} \circ \mathcal{T}: S_{m} \rightarrow S_{m}$ is weak-weak continuous. Since $S_{m}$ is obviously convex and compact in the weak topology of the space $L^{2}(0, T ; H)$, from the Schauder fixed point theorem it follows that there exists a fixed point $u_{n} \in S_{m}$ of the operator $\alpha_{n} \circ \mathcal{T}$, i.e.

$$
u_{n}=\alpha_{n}\left(\mathcal{T}\left(u_{n}\right)\right) .
$$

Setting $\left(\sigma_{n}, v_{n}, w_{n}\right):=\mathcal{T}\left(u_{n}\right)$, from (4.7)-(4.10) we see that $\left(\sigma_{n}, v_{n}, w_{n}, u_{n}\right) \in \mathcal{R}_{\mathcal{U}}$, $n \geq 1$, and

$$
\begin{aligned}
& \left|\gamma_{n}(t)-u_{n}(t)\right|_{H}^{2} \\
& \quad \leq \frac{2}{n^{2}}+12 k^{2}(t)\left(\left|\sigma_{*}(t)-\sigma_{n}(t)\right|_{H}^{2}+\left|v_{*}(t)-v_{n}(t)\right|_{H}^{2}+\left|w_{*}(t)-w_{n}(t)\right|_{H}^{2}\right) .
\end{aligned}
$$


Since on the set $S_{m}$ the topology induced by the "weak" norm in (4.5) coincides with the weak topology of the space $L^{2}(0, T ; H)$, from (4.5) we obtain

$$
\gamma_{n} \rightarrow u_{*} \quad \text { weakly in } L^{2}(0, T ; H) .
$$

Theorem 3.2 implies then that

$$
\left(\sigma\left(\gamma_{n}\right), v\left(\gamma_{n}\right), w\left(\gamma_{n}\right)\right) \rightarrow\left(\sigma_{*}, v_{*}, w_{*}\right) \quad \text { strongly in } C\left([0, T] ; H^{3}\right) .
$$

From (4.11) and (3.20) it follows that

$$
\begin{aligned}
& \mid \sigma_{n}(t)-\left.\sigma\left(\gamma_{n}\right)(t)\right|_{H} ^{2}+\left|v_{n}(t)-v\left(\gamma_{n}\right)(t)\right|_{H}^{2}+\left|w_{n}(t)-w\left(\gamma_{n}\right)(t)\right|_{H}^{2} \\
& \leq C_{m} \int_{0}^{t} \mid u_{n}(\tau)-\left.\gamma_{n}(\tau)\right|_{H} ^{2} d \tau \\
& \leq C_{m} \int_{0}^{t}\left(\frac{2}{n^{2}}+24 k^{2}(\tau)\left(\left|\sigma_{n}(\tau)-\sigma\left(\gamma_{n}\right)(\tau)\right|_{H}^{2}+\left|v_{n}(\tau)-v\left(\gamma_{n}\right)(\tau)\right|_{H}^{2}\right.\right. \\
&\left.\quad+\left|w_{n}(\tau)-w\left(\gamma_{n}\right)(\tau)\right|_{H}^{2}\right)+24 k^{2}(\tau)\left(\left|\sigma\left(\gamma_{n}\right)(\tau)-\sigma_{*}(\tau)\right|_{H}^{2}\right. \\
&\left.\left.\quad+\left|v\left(\gamma_{n}\right)(\tau)-v_{*}(\tau)\right|_{H}^{2}+\left|w\left(\gamma_{n}\right)(\tau)-w_{*}(\tau)\right|_{H}^{2}\right)\right) d \tau .
\end{aligned}
$$

Invoking Gronwall's lemma, from the last inequality and (4.13) we obtain

$$
\left(\sigma_{n}, v_{n}, w_{n}\right) \rightarrow\left(\sigma_{*}, v_{*}, w_{*}\right) \quad \text { strongly in } C\left([0, T] ; H^{3}\right) .
$$

Finally, from (4.11), (4.12), and Lebesgue's dominated convergence theorem we conclude that

$$
u_{n} \rightarrow u_{*} \quad \text { weakly in } L^{2}(0, T ; H),
$$

and the claim of Theorem 2.1 follows.

Set now $\mathcal{R}:=\left\{(\sigma(t), v(t), w(t)) ; t \in[0, T],(\sigma, v, w) \in \mathcal{R}_{\mathcal{U}}\right\}$. Theorem 3.2 together with Hypothesis (U3) imply that the set $\mathcal{R}$ is compact in $H^{3}$. Define $\varphi:[0, T] \times H^{3} \times H \rightarrow \mathbb{R} \cup\{+\infty\}$ by

$$
\varphi(t, \sigma, v, w, u)= \begin{cases}\mathfrak{g}_{\mathcal{U}}^{* *}(t, \sigma, v, w, u), & \text { if } t \in[0, T],(\sigma, v, w) \in \mathcal{R}, u \in H, \\ +\infty, & \text { otherwise. }\end{cases}
$$

By the property of $\mathfrak{g}_{\mathcal{U}}^{* *}$ stated after (4.4), there exists a sequence of closed sets $T_{n} \subset T_{n+1} \subset \cdots \subset[0, T], n \geq 1$, with $\mu\left([0, T] \backslash \bigcup_{n=1}^{\infty} T_{n}\right)=0$ such that the function $\mathfrak{g}_{\mathcal{U}}^{* *}(t, v, w, u)$ restricted to $T_{n} \times \mathcal{R} \times H, n \geq 1$, is lower semicontinuous. Since the set $\bigcup_{n=1}^{\infty} T_{n}$ is a Borel, the function $\varphi$ is Borel on $\bigcup_{n=1}^{\infty} T_{n} \times \mathcal{R} \times H$. Letting the values of $\varphi$ to be zero outside the set $\bigcup_{n=1}^{\infty} T_{n} \times \mathcal{R} \times H$, we can assume that $\varphi$ is Borel on $[0, T] \times \mathcal{R} \times H$ and, thus, it is measurable. From the fact that $\mathfrak{g}_{\mathcal{U}}^{* *}$ is the largest convex function such that $\mathfrak{g}_{\mathcal{U}}^{* *} \leq \mathfrak{g}$ and from $(\mathcal{U} \mathbf{3}),(\mathfrak{q} 3)$, (4.4) it follows that

$$
-l(t) \leq q(t, \sigma, v, w, u) \quad \text { for a.e. } t \in[0, T], \sigma, v, w, u \in H,
$$


for some $l \in L^{1}\left([0, T], \mathbb{R}^{+}\right)$.

Defining now the integral functional

$$
(v, w, u) \rightarrow J_{\varphi}(\sigma, v, w, u):=\int_{0}^{T} \varphi(t, \sigma(t), v(t), w(t), u(t)) d t
$$

from [36, Theorem 2.1] we deduce that $J_{\varphi}$ is sequentially lower semicontinuous on the space $C\left([0, T], H^{3}\right) \times L^{2}([0, T], H)$, when $L^{2}([0, T], H)$ is endowed with the weak topology. According to Theorem 3.2 the set $\mathcal{R}_{\overline{\mathrm{co}} \mathcal{U}}$ is compact in this space. Since, obviously, $J_{\varphi}(\sigma, v, w, u)=J_{U}^{* *}(\sigma, v, w, u)$ on $\mathcal{R}_{\overline{\mathrm{co}} \mathcal{U}}$, we conclude that problem $(R P)$ has a solution $\left(\sigma_{*}, v_{*}, w_{*}, u_{*}\right) \in \mathcal{R}_{\overline{\mathrm{co}} \mathcal{U}}$. Then, Theorem 2.1 implies that there exists a sequence $\left(\sigma_{n}, v_{n}, w_{n}, u_{n}\right) \in \mathcal{R}_{\mathcal{U}}, n \geq 1$, such that (2.5) (2.7) hold. In particular,

$$
J_{U}^{* *}\left(\sigma_{*}, v_{*}, w_{*}, u_{*}\right)=\lim _{n \rightarrow \infty} J\left(\sigma_{n}, v_{n}, w_{n}, u_{n}\right),
$$

which combined with

$$
J_{U}^{* *}\left(\sigma_{*}, v_{*}, w_{*}, u_{*}\right) \leq J_{U}^{* *}\left(\sigma_{n}, v_{n}, w_{n}, u_{n}\right) \leq J\left(\sigma_{n}, v_{n}, w_{n}, u_{n}\right)
$$

proves Theorem 2.2 .

\section{References}

[1] A. Visintin, Differential Models of Hysteresis. Appl. Math. Sci. 111, SpringerVerlag, Berlin, 1994.

[2] P. Colli, N. Kenmochi, and M. Kubo, A phase field model with temperature dependent constraint. J. Math. Anal. Appl. 256 (2001), 668-685.

[3] P. Krejčí, J. Sprekels, and U. Stefanelli, Phase-field models with hysteresis in one-dimensional thermoviscoplasticity. SIAM J. Math. Anal. 34 (2002), 409434.

[4] C. Giorgi, Phase-field models for transition phenomena in materials with hysteresis. Discrete Contin. Dyn. Syst. Ser. S 8 (2015), no. 4, 693-722.

[5] M. Helmers and M. Herrmann, Hysteresis and phase transitions in a lattice regularization of an ill-posed forward-backward diffusion equation. Arch. Ration. Mech. Anal. 230 (2018), no. 1, 231-275.

[6] A. Corli and H. Fan, Two-phase flow in porous media with hysteresis. J. Differential Equations 265 (2018), no. 4, 1156-1190.

[7] P. Krejčí, S.A. Timoshin, and A.A. Tolstonogov, Relaxation and optimisation of a phase-field control system with hysteresis, Int. J. Control 91 (2018), no. 1, $85-100$.

[8] M. Kubo, A filtration model with hysteresis. J. Differential Equations 201 (2004), 75-98. 
[9] P. Krejčí, J. P. O'Kane, A. Pokrovskii, and D. Rachinskii, Properties of solutions to a class of differential models incorporating Preisach hysteresis operator, Physica D 241 (2012), 2010-2028.

[10] B. Albers, Modeling the hysteretic behavior of the capillary pressure in partially saturated porous media: a review, Acta Mech. 225 (2014), no. 8, 2163-2189.

[11] B. Detmann, P. Krejčí, and E. Rocca, Solvability of an unsaturated porous media flow problem with thermomechanical interaction, SIAM J. Math. Anal. 48 (2016), no. 6, 4175-4201.

[12] P. Krejčí and S.A. Timoshin, Coupled ODEs control system with unbounded hysteresis region, SIAM J. Control Optim. 54 (2016), no. 4, 1934-1949.

[13] B. Cahlon, D. Schmidt, M. Shillor, and X. Zou, Analysis of thermostat models, Eur. J. Appl. Math. 8 (1997), no. 5, 437-455.

[14] J. Kopfová and T. Kopf, Differential equations, hysteresis, and time delay, Z. Angew. Math. Phys. 53 (2002), no. 4, 676-691.

[15] H. Logemann, E.P. Ryan, and I. Shvartsman, A class of differential-delay systems with hysteresis: Asymptotic behaviour of solutions, Nonlinear Anal. 69 (2008), no. 1, 363-391.

[16] P. Gurevich and E. Ron, Stability of periodic solutions for hysteresis-delay differential equations, J. Dynam. Differential Equations 31 (2019), no. 4, 18731920.

[17] S.A. Timoshin, Bang-bang control of a thermostat with nonconstant cooling power, ESAIM, Control Optim. Calc. Var. 24 (2018), no. 2, 709-719.

[18] T. Aiki and K. Kumazaki, Mathematical model for hysteresis phenomenon in moisture transport of concrete carbonation process, Phys. B 407 (2012), 14241426.

[19] T. Aiki and K. Kumazaki, Uniqueness of solutions to a mathematical model describing moisture transport in concrete materials, Netw. Heterog. Media 9 (2014), no. 4, 683-707

[20] M.M. Jensen, B. Johannesson, and M.R. Geiker, A numerical comparison of ionic multi-species diffusion with and without sorption hysteresis for cementbased materials, Transp. Porous Media 107 (2015), no. 1, 27-47.

[21] T. Aiki and S.A. Timoshin, Existence and uniqueness for a concrete carbonation process with hysteresis, J. Math. Anal. Appl. 449 (2017), no. 2, 1502-1519.

[22] S.A. Timoshin and T. Aiki, Extreme solutions in control of moisture transport in concrete carbonation, Nonlinear Anal., Real World Appl. 47 (2019), 446459 .

[23] T. Aiki and E. Minchev, A prey-predator model with hysteresis effect, SIAM J. Math. Anal. 36 (2005), no. 6, 2020-2032. 
[24] J. Zheng and Y. Wang, Well-posedness for a class of biological diffusion models with hysteresis effect, Z. Angew. Math. Phys. 66 (2015), no. 3, 771-783.

[25] Y. Wang and J. Zheng, Periodic solutions to a class of biological diffusion models with hysteresis effect, Nonlinear Anal. Real World Appl. 27 (2016), 297-311.

[26] S.A. Timoshin and T. Aiki, Control of biological models with hysteresis, Systems Control Lett. 128 (2019), 41-45.

[27] M. Brokate, Optimal control of systems described by ordinary differential equations with nonlinear characteristics of hysteresis type. [I], Automat. Remote Control 52 (1991), no. 12, part 1, 1639-1681.

[28] M. Brokate, Optimal control of systems described by ordinary differential equations with nonlinear characteristics of hysteresis type. [II], Automat. Remote Control 53 (1992), no. 1, part 1, 1-33.

[29] H. Brézis, Opérateurs Maximaux Monotones et Semi-groupes de Contractions dans les Espaces de Hilbert, North-Holland, Amsterdam, 1973.

[30] U. Mosco, Convergence of convex sets and of solutions of variational inequalities. Adv. Math. 3 (1969), 510-585.

[31] N. Kenmochi, Solvability of nonlinear evolution equations with time-dependent constraints and applications, Bull. Fac. Educ., Chiba Univ., Part 2, 30 (1981), $1-87$.

[32] F. Hiai and H. Umegaki, Integrals, conditional expectations, and martingales of multivalued functions, J. Multivariate Anal., 7 (1977), 149-182.

[33] F.S. De Blasi, G. Pianigiani, and A.A. Tolstonogov, A Bogolyubov-type theorem with a nonconvex constraint in Banach spaces, SIAM J. Control Optim. 43 (2004), no. 2, 466-476.

[34] Phan Van Chuong, A density theorem with an application in relaxation of nonconvex-valued differential equations. J. Math. Anal. Appl. 124 (1987), 1-14.

[35] A. Fryszkowski, Continuous selections for a class of nonconvex multivalued maps. Studia Math. 76 (1983), 163-174.

[36] E.J. Balder, Necessary and sufficient conditions for $L_{1}$-strong-weak lower semicontinuity of integral functionals. Nonlinear Anal., 11 (1987), no. 12, 13991404 . 